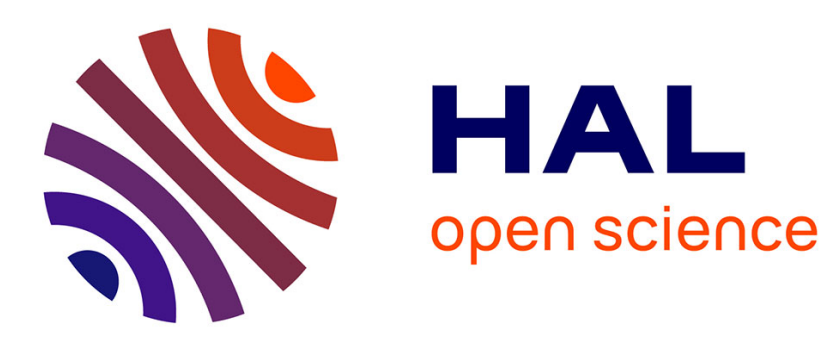

\title{
Parameter Estimation for the Square-root Diffusions : Ergodic and Nonergodic Cases
}

\author{
Mohamed Ben Alaya, Ahmed Kebaier
}

\section{To cite this version:}

Mohamed Ben Alaya, Ahmed Kebaier. Parameter Estimation for the Square-root Diffusions : Ergodic and Nonergodic Cases. 2010. hal-00579644

\section{HAL Id: hal-00579644 \\ https://hal.science/hal-00579644}

Preprint submitted on 24 Mar 2011

HAL is a multi-disciplinary open access archive for the deposit and dissemination of scientific research documents, whether they are published or not. The documents may come from teaching and research institutions in France or abroad, or from public or private research centers.
L'archive ouverte pluridisciplinaire HAL, est destinée au dépôt et à la diffusion de documents scientifiques de niveau recherche, publiés ou non, émanant des établissements d'enseignement et de recherche français ou étrangers, des laboratoires publics ou privés. 


\title{
PARAmeter Estimation for the SquARE-Root Diffusions : ERgodic And Nonergodic CASES
}

\author{
By Mohamed Ben Alaya and Ahmed Kebaier \\ LAGA, CNRS (UMR 7539), Institut Galilée, Université Paris 13, \\ 99, av. J.B. Clément 93430 Villetaneuse, France \\ mba@math.univ-paris13.fr kebaier@math.univ-paris13.fr
}

March 24, 2011

\begin{abstract}
This paper deals with the problem of parameter estimation in the Cox-Ingersoll-Ross (CIR) model $\left(X_{t}\right)_{t \geq 0}$. This model is frequently used in finance for example as a model for computing the zero-coupon bound price or as a dynamic of the volatility in the Heston model. When the diffusion parameter is known, the maximum likelihood estimator (MLE) of the drift parameters involves the quantities : $\int_{0}^{t} X_{s} d s$ and $\int_{0}^{t} \frac{d s}{X_{s}}$. At first, we study the asymptotic behavior of these processes. This allows us to obtain various and original limit theorems on our estimators, with different rates and different types of limit distributions. Our results are obtained for both cases : ergodic and nonergodic diffusion. Numerical simulations were processed using an exact simulation algorithm.

AMS 2000 Mathematics Subject Classification. 44A10, 60F05, 62F12, 65C05. Key Words and Phrases. Cox-Ingersoll-Ross processes, nonergodic diffusion, Laplace transform, limit theorems, parameter inference, simulation efficiency : exact methods.
\end{abstract}

\section{Introduction}

Over the last few years, an interesting process emerged and became quite popular in finance, after Cox-Ingersoll-Ross (CIR) proposed it for modelling short-term interest rates [4]. It is also used for modelling stochastic volatility in the Heston model [10]. The CIR process $\left(X_{t}\right)_{t \geq 0}$, also known as the square root diffusion, is solution to the stochastic differential equation (SDE)

$$
d X_{t}=\left(a-b X_{t}\right) d t+\sqrt{2 \sigma\left|X_{t}\right|} d W_{t}
$$

where $X_{0}=x>0, a>0, b \in \mathbb{R}, \sigma>0$ and $\left(W_{t}\right)_{t \geq 0}$ is a standard Brownian motion. Under the above assumption on the parameters that we will suppose valid through all the paper, this SDE has a unique strong solution $\left(X_{t}\right)_{t \geq 0}$ (see Ikeda and Watanabe [11], p. 221) and from the comparison theorem for one-dimensional diffusion process (see Revuz and Yor [20], p. 394) 
we deduce that $X_{t} \geq 0$. In the particular case $b=0$ and $\sigma=2$, we recover the square of a $a$-dimensional Bessel process starting at $x$. Let us recall now some basic properties on the CIR model and let $\tau_{0}:=\inf \left\{t \geq 0 \mid X_{t}=0\right\}$, with the convention $\inf \emptyset=\infty$. In the case $a \geq \sigma$, the process is strictly positive, $\tau_{0}$ is infinite almost surely, otherwise it is nonnegative, which means that it can reach the state 0 . More precisely, for $a<\sigma$ and $b \geq 0, \tau_{0}$ is finite almost surely and for $a<\sigma$ and $b<0$, we have $\left.\mathbb{P}_{x}\left(\tau_{0}<\infty\right) \in\right] 0,1[$. Note that in the case $0<a<\sigma$, when the process reaches the boundary, the state 0 is instantaneously reflecting (see e.g. Göing-Jaeschke and Yor [8] or Lamberton and Lapeyre [16] for more details). From the ergodicity point of view, the CIR process is ergodic and its stationary distribution, say $\pi$, is a Gamma law with shape parameter $a / \sigma$ and scale parameter $\sigma / b$, provided that $b>0$. In this case, for all $h \in L^{1}(\pi)$, $\frac{1}{t} \int_{0}^{t} h\left(X_{s}\right) d s$ converges almost surely to $\int_{\mathbb{R}} h(x) \pi(d x)$.

During the last decades, several authors studied the problem of estimating parameters in the drift coefficient when a diffusion process was observed continuously; this corresponds to observe the path of the diffusion over an interval $[0, T], T>0$. This theory has been established mainly by Lipster and Shiryayev [17] and Kutoyants [15]. This approach is rather theoretical, since the real data are discrete time observations. However, if the error due to discretization is negligible then the statistical results obtained for the continuous time model are valid for discrete time observations too. In the literature, most of the articles are concerned with ergodic diffusions and only few results can be found for the nonergodic case (see e.g [15] section 3.5 of chapter 3 and references there). In this last reference, many technics are proposed to construct estimators of the drift parameters. Furthermore, when the drift coefficient depends linearly on the parameters, one can hope to obtain a nice explicit formula of the maximum likelihood estimator (MLE). To our knowledge, one of the first papers having studied the MLE for the problem of estimating parameters in the CIR model is that of Fournié and Talay [7]. They have established its asymptotic normality in the case $b>0$ and $a>\sigma$.

The aim of this paper is to investigate the MLE of the drift parameters in the CIR model for a range of values $(a, b, \sigma)$ covering ergodic and nonergodic situations. Roughly speaking, if we estimate one of the drift parameters and suppose known the other one, the MLE error has the form $M_{t} /\langle M\rangle_{t}$, where $\left(M_{t}\right)_{t \geq 0}$ is a Brownian martingale with quadratic variation $\langle M\rangle_{t}$. If $b>0$ and $a>\sigma$, the asymptotic normality of the estimators is obtained using the classical martingale central limit theorem as in Fournié and Talay [7]. Otherwise, this argument is no more valid, even in the special ergodic case $b>0$ and $a \leq \sigma$, since $\int_{\mathbb{R}}(1 / x) \pi(d x)=\infty$. To overcome this difficulty, we study the asymptotic behavior of the couple $\left(M_{t},\langle M\rangle_{t}\right)$.

In the second section, as in our framework $\langle M\rangle_{t}$ is either $\int_{0}^{t} X_{s} d s$ or $\int_{0}^{t} \frac{d s}{X_{s}}$, we proceed by computing their Laplace transform. The first one is well known (see e.g. Lamberton and Lapeyre [16], p. 127). However for the second one, which is more subtle, we apply recent results of Craddock and Lennox [5], who employ Lie symmetry methods to evaluate certain expectations for a large class of Itô diffusions. This allows us to obtain a precise description of the asymptotic of the $\int_{0}^{t} X_{s} d s$ (see Proposition 1 and 3 ) and $\int_{0}^{t} \frac{d s}{X_{s}}$ (see Proposition 2 and 4).

Then in the third section, we take advantage of this study to prove new original results on the asymptotic of the MLE that are not necessarily normal. The asymptotic theorem concerning the MLE, $\hat{b}_{T}$ (resp. $\hat{a}_{T}$ ), of $b$ (resp. $a$ ) is obtained with different rates of convergence that are unusual in most cases : $\sqrt{T}$ for $b>0, T$ for $b=0$ and $e^{-b T / 2}$ for $b<0$ (resp. $\sqrt{T}$ for $b>0$ and $a>\sigma, T$ for $b>0$ and $a=\sigma, \sqrt{\log T}$ for $b=0$ and $a>\sigma$ and $\log T$ for $b=0$ and $a=\sigma$ ). 
In those different cases, the corresponding limit distributions are given by Theorem 1 for the MLE of $b$ and Theorem 2 for the MLE of $a$.

Finally in the last section, we illustrate our asymptotic results using an exact simulation method. Indeed, to simulate the couple $\left(X_{T}, \int_{0}^{T} X_{s} d s\right)$ we use and perfect the method of Broadie and Kaya [3] based on an explicit evaluation of the conditional Laplace transform of $\int_{0}^{T} X_{s} d s$ given $X_{T}$. Concerning $\left(X_{T}, \int_{0}^{T} \frac{d s}{X_{s}}\right)$, we first establish a new explicit formula of the conditional Laplace transform of $\int_{0}^{T} \frac{d s}{X_{s}}$ given $X_{T}$ (see Theorem 3 and 4 ) and then we deduce an exact simulation method of the couple in the same manner as in [3].

\section{The Asymptotic Behavior of $\int_{0}^{t} X_{s} d s$ and $\int_{0}^{t} \frac{d s}{X_{s}}$}

Let us recall that $\left(X_{t}\right)_{t \geq 0}$ denotes a CIR process solution to (1). It is relevant to consider separately the cases $b=0$ and $b \neq 0$, since the process $\left(X_{t}\right)_{t \geq 0}$ behaves differently.

\subsection{Case $b=0$}

In this section, we consider the Cox-Ingersoll-Ross CIR process $\left(X_{t}\right)_{t \geq 0}$ with $b=0$. In this particular case, $\left(X_{t}\right)_{t \geq 0}$ satisfies the $\mathrm{SDE}$

$$
d X_{t}=a d t+\sqrt{2 \sigma X_{t}} d W_{t}
$$

Note that for $\sigma=2$, we recover the square of a $a$-dimensional Bessel process starting at $x$ and denoted by $\mathrm{BESQ}_{x}^{a}$. This process has been attracting considerably the attention of several studies (see Revuz and Yor [20]). The asymptotic behavior of $\left(X_{t}, \int_{0}^{t} X_{s} d s\right)$ and $\int_{0}^{t} \frac{d s}{X_{s}}$ are established by our next two propositions.

Proposition 1 Let $\left(X_{t}\right)_{t \geq 0}$ be a CIR process solution to (2), we have

$$
\left(\frac{X_{t}}{t}, \frac{1}{t^{2}} \int_{0}^{t} X_{s} d s\right) \stackrel{\text { law }}{\longrightarrow}\left(R_{1}, I_{1}\right) \text { as } t \text { tends to infinity. }
$$

where $\left(R_{t}\right)_{t \geq 0}$ is the CIR process starting from 0 , solution to (2) and $I_{t}=\int_{0}^{t} R_{s} d s$.

Proposition 2 Under the above notations, we have

1. $\mathbb{P}_{x}\left(\int_{0}^{t} \frac{d s}{X_{s}}<\infty\right)=1$ if and only if $a \geq \sigma$.

2. If $a>\sigma$ then $\frac{1}{\log t} \int_{0}^{t} \frac{d s}{X_{s}} \stackrel{\mathbb{P}}{\longrightarrow} \frac{1}{a-\sigma}$ as $t$ tends to infinity.

3. If $a=\sigma$ then $\frac{1}{(\log t)^{2}} \int_{0}^{t} \frac{d s}{X_{s}} \stackrel{\text { law }}{\longrightarrow} \tau_{1}$ as $t$ tends to infinity, where $\tau_{1}$ is the hitting time associated with Brownian motion $\tau_{1}:=\inf \left\{t>0: W_{t}=\frac{1}{\sqrt{2 \sigma}}\right\}$. 
In order to prove these propositions, we choose to compute the Laplace transform of the couple $\left(X_{t}, \int_{0}^{t} X_{s} d s\right)$ and $\int_{0}^{t} \frac{d s}{X_{s}}$. Here are the obtained results.

Lemma 1 We have

- For $\lambda \geq 0$ and $\mu \geq 0$,

$$
\mathbb{E}_{x}\left(e^{-\lambda X_{t}-\mu \int_{0}^{t} X_{s} d s}\right)=e^{-a \phi_{\lambda, \mu}(t)} e^{-x \psi_{\lambda, \mu}(t)},
$$

where functions $\phi_{\lambda, \mu}$ and $\psi_{\lambda, \mu}$ are given by

$$
\phi_{\lambda, \mu}(t)=\frac{1}{\sigma} \log \left(\frac{2 \sigma \lambda}{\rho} \sinh (\rho t / 2)+\cosh (\rho t / 2)\right), \psi_{\lambda, \mu}(t)=\frac{\lambda \cosh (\rho t / 2)+\frac{2 \mu}{\rho} \sinh (\rho t / 2)}{\frac{2 \sigma \lambda}{\rho} \sinh (\rho t / 2)+\cosh (\rho t / 2)}
$$

and $\rho=2 \sqrt{\sigma \mu}$.

- For $\mu>0$,

$$
\mathbb{E}_{x}\left(e^{-\mu \int_{0}^{t} \frac{d s}{X_{s}}}\right)=\frac{\Gamma\left(k+\frac{\nu}{2}+\frac{1}{2}\right)}{\Gamma(\nu+1)}\left(\frac{x}{\sigma t}\right)^{\frac{\nu}{2}+\frac{1}{2}-k} \exp \left(-\frac{x}{\sigma t}\right){ }_{1} F_{1}\left(k+\frac{\nu}{2}+\frac{1}{2}, \nu+1, \frac{x}{\sigma t}\right),
$$

where $k=\frac{a}{2 \sigma}, \nu=\frac{1}{\sigma} \sqrt{(a-\sigma)^{2}+4 \mu \sigma}$ and ${ }_{1} F_{1}$ is the confluent hypergeometric function defined by ${ }_{1} F_{1}(u, v, z)=\sum_{n=0}^{\infty} \frac{u_{n}}{v_{n}} \frac{z^{n}}{n !}$, with $u_{0}=v_{0}=1$, and for $n \geq 1, u_{n}=\prod_{k=0}^{n-1}(u+k)$ and $v_{n}=\prod_{k=0}^{n-1}(v+k)$.

Proof : Taking $b=0$ in Proposition 2.5 of chapter 6 in [16], we deduce the first assertion. For the second one, we apply Theorem 5.10 in [5] to our process. We have just to be careful with the misprint in formula (5.24) of [5]. More precisely, we have to replace $\sqrt{A x y}$, in the numerator of the first term in the right hand side of this formula, by $\sqrt{A x / y}$. Hence, for $a>0$ and $\sigma>0$, we obtain the so called fundamental solution of the PDE $u_{t}=\sigma x u_{x x}+a u_{x}-\left(\frac{\mu}{x}+\lambda x\right) u, \quad \lambda>$ $0, \mu>0$ :

$$
p(t, x, y)=\frac{\sqrt{\sigma \lambda}}{\sigma \sinh (\sqrt{\sigma \lambda} t)}\left(\frac{y}{x}\right)^{k-1 / 2} \exp \left(-\frac{\sqrt{\sigma \lambda}(x+y)}{\sigma \tanh (\sqrt{\sigma \lambda} t)}\right) I_{\nu}\left(\frac{2 \sqrt{\sigma \lambda} \sqrt{x y}}{\sigma \sinh (\sqrt{\sigma \lambda} t)}\right),
$$

where $I_{\nu}$ is the modified Bessel function of the first kind. This yields the Laplace transform of the couple $\left(\int_{0}^{t} X_{s} d s, \int_{0}^{t} \frac{d s}{X_{s}}\right)$, since $\mathbb{E}_{x}\left(e^{-\lambda \int_{0}^{t} X_{s} d s-\mu \int_{0}^{t} \frac{d s}{X_{s}}}\right)=\int_{0}^{\infty} p(t, x, y) d y$. Evaluation of this integral is routine, see formula 2 of section 6.643 in [9]. Therefore, we get

$$
\begin{array}{r}
\mathbb{E}_{x}\left(e^{-\lambda \int_{0}^{t} X_{s} d s-\mu \int_{0}^{t} \frac{d s}{X_{s}}}\right)=\frac{\Gamma\left(k+\frac{\nu}{2}+\frac{1}{2}\right)}{\Gamma(\nu+1)}\left(\frac{\sqrt{\sigma \lambda} x \operatorname{coth}(\sqrt{\sigma \lambda} t)}{\sigma}\right)^{-k} \exp \left(-\frac{\sqrt{\sigma \lambda} x}{\sigma} \operatorname{coth}(\sqrt{\sigma \lambda} t)\right) \\
\times \exp \left(\frac{\sqrt{\sigma \lambda} x}{2 \sigma \sinh (\sqrt{\sigma \lambda} t) \cosh (\sqrt{\sigma \lambda} t)}\right) M_{-k, \frac{\nu}{2}}\left(\frac{\sqrt{\sigma \lambda} x}{\sigma \sinh (\sqrt{\sigma \lambda} t) \cosh (\sqrt{\sigma \lambda} t)}\right), \quad(5)
\end{array}
$$


where $M_{s, r}(z)$ is the Whittaker function of the first kind given by

$$
M_{s, r}(z)=z^{r+\frac{1}{2}} e^{-\frac{z}{2}}{ }_{1} F_{1}\left(r-s+\frac{1}{2}, 2 r+1, z\right)
$$

See [9] for more details about those special functions. By inserting relation (6) in (5) we obtain

$$
\begin{aligned}
& \mathbb{E}_{x}\left(e^{-\lambda \int_{0}^{t} X_{s} d s-\mu \int_{0}^{t} \frac{d s}{X_{s}}}\right)=\frac{\Gamma\left(k+\frac{\nu}{2}+\frac{1}{2}\right)}{\Gamma(\nu+1)}(\cosh (\sqrt{\sigma \lambda} t))^{-\frac{\nu}{2}-\frac{1}{2}-k}\left(\frac{\sqrt{\sigma \lambda} x}{\sigma \sinh (\sqrt{\sigma \lambda} t)}\right)^{\frac{\nu}{2}+\frac{1}{2}-k} \\
& \quad \times \exp \left(-\frac{\sqrt{\sigma \lambda} x}{\sigma} \operatorname{coth}(\sqrt{\sigma \lambda} t)\right){ }_{1} F_{1}\left(k+\frac{\nu}{2}+\frac{1}{2}, \nu+1, \frac{\sqrt{\sigma \lambda} x}{\sigma \sinh (\sqrt{\sigma \lambda} t) \cosh (\sqrt{\sigma \lambda} t)}\right) .
\end{aligned}
$$

We complete the proof by letting $\lambda$ tend to 0 .

Proof of Proposition 1 : Under the notations of the above Lemma, it is easy to check that

$$
\phi_{\frac{\lambda}{t}, \frac{\mu}{t^{2}}}(t)=-\frac{1}{\sigma} \log \left(\frac{\sqrt{\sigma \mu}}{\sigma \lambda \sinh (\sqrt{\sigma \mu})+\sqrt{\sigma \mu} \cosh (\sqrt{\sigma \mu})}\right), \quad \lim _{t \rightarrow \infty} \psi_{\frac{\lambda}{t}, \frac{\mu}{t^{2}}}(t)=0
$$

and

$$
\lim _{t \rightarrow \infty} \mathbb{E}_{x}\left(e^{-\lambda \frac{X_{t}}{t}-\frac{\mu}{t^{2}} \int_{0}^{t} X_{s} d s}\right)=\left(\frac{\sqrt{\sigma \mu}}{\sigma \lambda \sinh (\sqrt{\sigma \mu})+\sqrt{\sigma \mu} \cosh (\sqrt{\sigma \mu})}\right)^{\frac{a}{\sigma}} .
$$

Noting that the first assertion of Lemma 1 remains valid with $x=0$ (see [16]), we deduce that the obtained limit is simply the Laplace transform of the CIR process starting from 0 , solution to (2) with $t=1$. This completes the proof.

Remark It is worth to note that Proposition 1 can be obtained by a scaling argument, but in order to standardize the technics used in this section we dropped this idea.

Proof of Proposition 2 For $a \geq \sigma$, by Lemma 1, we have

$$
\mathbb{P}_{x}\left(\int_{0}^{t} \frac{d s}{X_{s}}<\infty\right)=\lim _{\mu \rightarrow \infty} \mathbb{E}_{x}\left(e^{-\mu \int_{0}^{t} \frac{d s}{X_{s}}}\right)=1
$$

In the case $a<\sigma$, we have

$$
\mathbb{P}_{x}\left(\int_{0}^{t} \frac{d s}{X_{s}}<\infty\right)=\frac{1}{\Gamma\left(2-\frac{a}{\sigma}\right)}\left(\frac{x}{\sigma t}\right)^{1-\frac{a}{\sigma}} \exp \left(-\frac{x}{\sigma t}\right){ }_{1} F_{1}\left(1,2-\frac{a}{\sigma}, \frac{x}{\sigma t}\right) .
$$

Thanks to the following formula (see section 9.211 in [9])

$$
{ }_{1} F_{1}(r, s, z)=\frac{\Gamma(s)}{\Gamma(r) \Gamma(s-r)} z^{1-s} \int_{0}^{z} e^{u} u^{r-1}(z-u)^{s-r-1} d u
$$


for $\operatorname{Re}(s)>\operatorname{Re}(r)>0$, we obtain

$$
\mathbb{P}_{x}\left(\int_{0}^{t} \frac{d s}{X_{s}}<\infty\right)=\frac{e^{-\frac{x}{\sigma t}}}{\Gamma\left(1-\frac{a}{\sigma}\right)} \int_{0}^{\frac{x}{\sigma t}} e^{u}\left(\frac{x}{\sigma t}-u\right)^{-\frac{a}{\sigma}} d u
$$

After the change of variable, $v=\frac{x}{\sigma t}-u$, the last relation becomes

$$
\mathbb{P}_{x}\left(\int_{0}^{t} \frac{d s}{X_{s}}<\infty\right)=\frac{1}{\Gamma\left(1-\frac{a}{\sigma}\right)} \int_{0}^{\frac{x}{\sigma t}} e^{-v} v^{-\frac{a}{\sigma}} d v<1 .
$$

For the second and the third assertions, we consider a positive function $\gamma(t)$ increasing to $+\infty$ when $t \rightarrow+\infty$. Using standard evaluations, it is easy to prove that

$$
\lim _{t \rightarrow+\infty} \mathbb{E}_{x}\left(e^{-\frac{\mu}{\gamma(t)^{2}} \int_{0}^{t} \frac{d s}{X_{s}}}\right)=\lim _{t \rightarrow+\infty} \exp \left(-\frac{1}{2 \sigma}\left(\sigma-a+\sqrt{(a-\sigma)^{2}+\frac{4 \mu \sigma}{\gamma(t)^{2}}}\right) \log (t)\right) .
$$

- If $a>\sigma$, let $\varepsilon$ denotes a function such that $\lim _{x \rightarrow 0} \varepsilon(x)=0$, we have

$$
\begin{aligned}
\lim _{t \rightarrow+\infty} \mathbb{E}_{x}\left(e^{-\frac{\mu}{\gamma(t)^{2}} \int_{0}^{t} \frac{d s}{X_{s}}}\right) & =\lim _{t \rightarrow+\infty} \exp \left(-\left(\frac{\mu}{\gamma(t)^{2}(a-\sigma)}+\frac{1}{\gamma(t)^{2}} \varepsilon\left(\frac{1}{\gamma(t)^{2}}\right) \log (t)\right)\right) \\
& =\exp \left(-\frac{\mu}{a-\sigma}\right), \text { by taking } \gamma(t)^{2}=\log (t) .
\end{aligned}
$$

- If $a=\sigma$

$$
\begin{aligned}
\lim _{t \rightarrow+\infty} \mathbb{E}_{x}\left(e^{-\frac{\mu}{\gamma(t)^{2}} \int_{0}^{t} \frac{d s}{X_{s}}}\right) & =\lim _{t \rightarrow+\infty} \exp \left(-\frac{1}{\sqrt{\sigma}} \frac{\sqrt{\mu}}{\gamma(t)} \log (t)\right) \\
& =\exp \left(-\frac{\sqrt{\mu}}{\sqrt{\sigma}}\right), \text { by taking } \gamma(t)=\log (t)
\end{aligned}
$$

This completes the proof.

We now turn to the case $b \neq 0$.

\section{$2.2 \quad$ Case $b \neq 0$}

Let us resume the general model of the CIR given by relation (1) with $b \neq 0$, namely

$$
d X_{t}=\left(a-b X_{t}\right) d t+\sqrt{2 \sigma X_{t}} d W_{t},
$$

where $X_{0}=x>0, a>0, b \in \mathbb{R}^{*}, \sigma>0$. Note that this process may be represented in terms of a square Bessel process through the relation $X_{t}=e^{-b t} Y\left(\frac{\sigma}{2 b}\left(e^{b t}-1\right)\right)$, where $Y$ denotes a BESQ $\frac{2 a}{\sigma}$. This relation results from simple properties of square Bessel processes (see e.g. Göing-Jaeschke and Yor [8] and Revuz and Yor [20]). We can now formulate the main results of this subsection. 
Proposition 3 Let $\left(X_{t}\right)_{t \geq 0}$ be a CIR process solution to (7), we have

1. If $b>0$ then $\frac{1}{t} \int_{0}^{t} X_{s} d s \stackrel{\mathbb{P}}{\longrightarrow} \frac{a}{b}$ as $t$ tends to infinity.

2. If $b<0$ then $\left(e^{b t} X_{t}, e^{b t} \int_{0}^{t} X_{s} d s\right) \stackrel{\text { law }}{\longrightarrow}\left(R_{t_{0}}, t_{0} R_{t_{0}}\right)$, as t tends to infinity, where $t_{0}=-1 / b$ and $\left(R_{t}\right)_{t \geq 0}$ is the CIR process, starting from $x$, solution to (2).

Proposition 4 Under the above notations, we have

1. $\mathbb{P}_{x}\left(\int_{0}^{t} \frac{d s}{X_{s}}<\infty\right)=1$ if and only if $a \geq \sigma$.

2. If $b>0$ and $a>\sigma$ then $\frac{1}{t} \int_{0}^{t} \frac{d s}{X_{s}} \stackrel{\mathbb{P}}{\longrightarrow} \frac{b}{a-\sigma}$ as $t$ tends to infinity.

3. If $b>0$ and $a=\sigma$ then $\frac{1}{t^{2}} \int_{0}^{t} \frac{d s}{X_{s}} \stackrel{\text { law }}{\longrightarrow} \tau_{2}$ as $t$ tends to infinity, where $\tau_{2}$ is the hitting time associated with Brownian motion $\tau_{2}:=\inf \left\{t>0: W_{t}=\frac{b}{\sqrt{2 \sigma}}\right\}$.

4. If $b<0$ and $a \geq \sigma$ then $\int_{0}^{t} \frac{d s}{X_{s}} \stackrel{\text { law }}{\longrightarrow} I_{t_{0}}:=\int_{0}^{t_{0}} R_{s} d s$ as tends to infinity, where $t_{0}=-1 / b$ and $\left(R_{t}\right)_{t \geq 0}$ is the CIR process, starting from $x$, solution to (2).

Remark : When $a>\sigma$ and $b>0$ the CIR process is ergodic and the stationary distribution is a Gamma law with shape $a / \sigma$ and scale $\sigma / b$. Let $\xi \stackrel{\text { law }}{=} \Gamma(a / \sigma, \sigma / b)$, according to the ergodic theorem, $\frac{1}{t} \int_{0}^{t} X_{s} d s \stackrel{\mathbb{P}-\text { p.s. }}{\longrightarrow} \mathbb{E}(\xi)=\frac{a}{b}$ and $\frac{1}{t} \int_{0}^{t} \frac{d s}{X_{s}} \stackrel{\mathbb{P}-p . s .}{\longrightarrow} \mathbb{E}\left(\frac{1}{\xi}\right)=\frac{b}{a-\sigma}$ as $t$ tends to infinity. In this case we recover the first assertion of Proposition 3 and the second assertion of Proposition 4.

In order to prove these propositions we need the following result.

Lemma 2 We have,

- for $\lambda \geq 0$ and $\mu \geq 0$, the Laplace transform of $\left(X_{t}, \int_{0}^{t} X_{s} d s\right)$ is given by

$$
\mathbb{E}_{x}\left(e^{-\lambda X_{t}-\mu \int_{0}^{t} X_{s} d s}\right)=e^{-a \tilde{\phi}_{\lambda, \mu}(t)} e^{-x \tilde{\psi}_{\lambda, \mu}(t)},
$$

where

$$
\tilde{\phi}_{\lambda, \mu}(t)=-\frac{1}{\sigma} \log \left(\frac{2 \rho e^{t(b-\rho) / 2}}{2 \sigma \lambda\left(1-e^{-\rho t}\right)+(\rho-b) e^{-\rho t}+(\rho+b)}\right)
$$

and

$$
\tilde{\psi}_{\lambda, \mu}(t)=\frac{\lambda\left((\rho+b) e^{-\rho t}+(\rho-b)\right)+2 \mu\left(1-e^{-\rho t}\right)}{2 \sigma \lambda\left(1-e^{-\rho t}\right)+(\rho-b) e^{-\rho t}+(\rho+b)},
$$

with $\rho=\sqrt{b^{2}+4 \sigma \mu}$. 
- For $\mu>0$, the Laplace transform of $\int_{0}^{t} \frac{d s}{X_{s}}$ is given by

$$
\begin{aligned}
\mathbb{E}_{x}\left(e^{-\mu \int_{0}^{t} \frac{d s}{X_{s}}}\right)= & \frac{\Gamma\left(k+\frac{\nu}{2}+\frac{1}{2}\right)}{\Gamma(\nu+1)} \frac{1}{x^{k} \alpha^{k}} \beta^{\frac{\nu}{2}+\frac{1}{2}} \\
& \times \exp \left(\frac{b}{2 \sigma}\left[a t-\frac{2 x}{e^{b t}-1}\right]\right){ }_{1} F_{1}\left(k+\frac{\nu}{2}+\frac{1}{2}, \nu+1, \beta\right),
\end{aligned}
$$

where $k=\frac{a}{2 \sigma}, \alpha=\frac{b e^{b t}}{\sigma\left(e^{b t}-1\right)}, \beta=\frac{b x}{\sigma\left(e^{b t}-1\right)}$ and $\nu=\frac{1}{\sigma} \sqrt{(a-\sigma)^{2}+4 \mu \sigma}$.

Remark : Note that the limit of the above Laplace transform, when $b$ goes to 0 , in formula (9) allows us to recover relation (3).

Proof The first assertion is given by Proposition 2.5 of chapter 6 in [16]. For the second one, we first apply Theorem 5.7 of [5], for $b \in \mathbb{R}$. We obtain the fundamental solution of the PDE $u_{t}=\sigma x u_{x x}+(a-b x) u_{x}-\frac{\mu}{x} u, \mu>0$ :

$$
\begin{aligned}
p(t, x, y)= & \frac{|b|}{2 \sigma \sinh (|b| t / 2)}\left(\frac{y}{x}\right)^{a /(2 \sigma)-1 / 2} \\
& \times \exp \left(\frac{b}{2 \sigma}[a t+(x-y)]-\frac{|b|(x+y)}{2 \sigma \tanh (|b| t / 2)}\right) I_{\nu}\left(\frac{|b| \sqrt{x y}}{\sigma \sinh (|b| t / 2)}\right) .
\end{aligned}
$$

By the parity of hyperbolic functions, we omit the $|$.$| in the above formula. This yields the$ Laplace transform of $\int_{0}^{t} \frac{d s}{X_{s}}$, since $\mathbb{E}_{x}\left(e^{-\mu \int_{0}^{t} \frac{d s}{X_{s}}}\right)=\int_{0}^{\infty} p(t, x, y) d y$. In the same manner as in the proof of Lemma 1, formula 2 of section 6.643 in [9] gives us

$$
\mathbb{E}_{x}\left(e^{-\mu \int_{0}^{t} \frac{d s}{X_{s}}}\right)=\frac{\Gamma\left(k+\frac{\nu}{2}+\frac{1}{2}\right)}{\Gamma(\nu+1)} \frac{e^{\frac{\beta}{2}}}{x^{k} \alpha^{k}} \exp \left(\frac{b}{2 \sigma}\left[a t-\frac{2 x}{e^{b t}-1}\right]\right) M_{-k, \frac{\nu}{2}}(\beta) .
$$

Finally, by inserting relation (6) in (11) we obtain the announced result.

Remark : In the above proof, relation (10) extends Corollary 5.8 of [5], established in the case $b>0$, to the case $b \in \mathbb{R}$. It is worth to note that formula (5.20) in this Corollary remains valid for this extension, thanks to the parity of hyperbolic functions.

In the following proofs $\varepsilon$ will denotes a function satisfying $\lim _{x \rightarrow 0} \varepsilon(x)=0$, that can change from an evaluation to an other.

Proof of Proposition 3 Let $\gamma(t)$ be a (non-random) positive function increasing to $+\infty$ when $t \rightarrow+\infty$, we take $\lambda=0$ and replace $\mu$ by $\mu / \gamma(t)^{2}$ in relation (8). In the case $b>0$, an easy computation shows that

$$
\begin{aligned}
\lim _{t \rightarrow+\infty} \mathbb{E}_{x}\left(e^{-\frac{\mu}{\gamma(t)^{2}} \int_{0}^{t} X_{s} d s}\right) & =\lim _{t \rightarrow+\infty} \exp \left(-\frac{a b}{2 \sigma} t\left(\sqrt{1+\frac{4 \mu \sigma}{b^{2} \gamma(t)^{2}}}-1\right)\right) \\
& =\exp \left(-\frac{a b}{2 \sigma} \frac{t}{\gamma(t)^{2}}\left(\frac{2 \mu \sigma}{b^{2}}+\varepsilon\left(\frac{1}{\gamma(t)^{2}}\right)\right)\right) .
\end{aligned}
$$


The first assertion follows by choosing $\gamma(t)^{2}=t$.

Next, we study the case $b<0$. According to the first assertion of Lemma 2, we have

$$
\mathbb{E}_{x}\left(e^{-\lambda e^{b t} X_{t}-\mu e^{b t} \int_{0}^{t} X_{s} d s}\right)=e^{-a \tilde{\phi}_{\lambda e}^{b t}, \mu e^{b t}(t)} e^{-x \tilde{\psi}_{\lambda e} e^{b t}, \mu e^{b t}(t)}
$$

with $\rho=\sqrt{b^{2}+4 \sigma \mu e^{b t}}$. As the Taylor's expansion of $\rho+b$ is equal to $-\frac{2 \sigma \mu}{b} e^{b t}+e^{b t} \varepsilon\left(e^{b t}\right)$, we deduce that $\lim _{t \rightarrow+\infty}(\rho+b) t=0$ and $\lim _{t \rightarrow+\infty}(\rho+b) e^{\rho t}=\lim _{t \rightarrow+\infty}(\rho+b) e^{-b t}=-\frac{2 \sigma \mu}{b}$. Hence, it's easy to check that

$$
\lim _{t \rightarrow+\infty} \tilde{\phi}_{\lambda e^{b t}, \mu e^{b t}}(t)=-\frac{1}{\sigma} \log \left(\frac{-b}{\sigma \lambda-b-\frac{\sigma}{b} \mu}\right) \quad \text { and } \quad \lim _{t \rightarrow+\infty} \tilde{\psi}_{\lambda e^{b t}, \mu e^{b t}}(t)=\frac{-b \lambda+\mu}{\sigma \lambda-b-\frac{\sigma}{b} \mu} .
$$

Therefore

$$
\lim _{t \rightarrow+\infty} \mathbb{E}_{x}\left(e^{-\lambda e^{b t} X_{t}-\mu e^{b t} \int_{0}^{t} X_{s} d s}\right)=\left(\frac{-b}{\sigma \lambda-b-\frac{\sigma}{b} \mu}\right)^{a / \sigma} \exp \left(-x \frac{-b \lambda+\mu}{\sigma \lambda-b-\frac{\sigma}{b} \mu}\right)
$$

In the other hand, using the first assertion of Lemma 3, we identify the Laplace transform of the announced couple limit $\left(R_{t_{0}}, t_{0} R_{t_{0}}\right)$, which completes the proof.

Remark : For $b<0$, we can also give this representation $e^{b t} \int_{0}^{t} X_{s} d s \stackrel{\text { law }}{\longrightarrow} L:=L 1+$ $L 2$ as $t$ tends to infinity where $L 1$ and $L 2$ are two independent random variables, $L 1$ has a gamma distribution and $L 2$ has a compound Poisson distribution. More precisely $L_{1} \stackrel{\text { law }}{=}$ $\Gamma\left(a / \sigma, \sigma / b^{2}\right)$ and $L_{2} \stackrel{\text { law }}{=} \sum_{k=1}^{N} X_{k}$ where $N \stackrel{\text { law }}{=} \mathcal{P}(x b / \sigma)$, for all $k \geq 1, X_{k} \stackrel{\text { law }}{=} \mathcal{E}\left(\sigma / b^{2}\right)$ and $\left(N, X_{1}, \cdots, X_{n}, \cdots\right)$ are mutually independent.

Proof of Proposition 4 Note that, the Laplace transform of $\int_{0}^{t} \frac{d s}{X_{s}}$ converges to $\mathbb{P}_{x}\left(\int_{0}^{t} \frac{d s}{X_{s}}<\right.$ $\infty)$ as $\mu \rightarrow 0$. If $a \geq \sigma$, using standard evaluations, it is easy to prove that

$$
\mathbb{P}_{x}\left(\int_{0}^{t} \frac{d s}{X_{s}}<\infty\right)=\left(\frac{\beta}{x \alpha}\right)^{\frac{a}{2 \sigma}} \exp \left(\frac{b}{2 \sigma}\left[a t-\frac{2 x}{e^{b t}-1}\right]+\beta\right)=1 .
$$

In the other case, $a<\sigma$, we have

$$
\begin{aligned}
\mathbb{P}_{x}\left(\int_{0}^{t} \frac{d s}{X_{s}}<\infty\right) & =\frac{1}{\Gamma\left(2-\frac{a}{\sigma}\right)} \frac{\beta^{1-\frac{a}{2 \sigma}}}{(x \alpha)^{\frac{a}{2 \sigma}}} \exp \left(\frac{b}{2 \sigma}\left[a t-\frac{2 x}{e^{b t}-1}\right]\right){ }_{1} F_{1}\left(1,2-\frac{a}{\sigma}, \beta\right) \\
& =\frac{\beta^{1-\frac{a}{\sigma}}}{\Gamma\left(2-\frac{a}{\sigma}\right)} e^{-\beta}{ }_{1} F_{1}\left(1,2-\frac{a}{\sigma}, \beta\right) .
\end{aligned}
$$

Thanks to the following formula (see section 9.211 in [9])

$$
{ }_{1} F_{1}(r, s, z)=\frac{\Gamma(s)}{\Gamma(r) \Gamma(s-r)} z^{1-s} \int_{0}^{z} e^{u} u^{r-1}(z-u)^{s-r-1} d u
$$


for $\operatorname{Re}(s)>\operatorname{Re}(r)>0$, we obtain

$$
\mathbb{P}_{x}\left(\int_{0}^{t} \frac{d s}{X_{s}}<\infty\right)=\frac{e^{-\beta}}{\Gamma\left(1-\frac{a}{\sigma}\right)} \int_{0}^{\beta} e^{u}(\beta-u)^{-\frac{a}{\sigma}} d u .
$$

After the change of variable, $v=\beta-u$, the last relation becomes

$$
\mathbb{P}_{x}\left(\int_{0}^{t} \frac{d s}{X_{s}}<\infty\right)=\frac{1}{\Gamma\left(1-\frac{a}{\sigma}\right)} \int_{0}^{\beta} e^{-v} v^{-\frac{a}{\sigma}} d v<1 .
$$

Now our task is to study the asymptotic behavior in distribution of the quantity $\int_{0}^{t} \frac{d s}{X_{s}}$ when $b>0$ and $a \geq \sigma$. Let $\gamma(t)$ be a (non-random) positive function increasing to $+\infty$ when $t \rightarrow+\infty$. If we replace $\mu$ by $\mu / \gamma(t)^{2}$ in relation $(9)$, since $\log (\beta)=\log (b x / \sigma)-b t-\log \left(1-e^{-b t}\right)$,

$$
\begin{aligned}
\lim _{t \rightarrow+\infty} \mathbb{E}_{x}\left(e^{-\frac{\mu}{\gamma(t)^{2}} \int_{0}^{t} \frac{d s}{X_{s}}}\right) & =\lim _{t \rightarrow+\infty} \frac{1}{x^{k} \alpha^{k}} \exp \left(\frac{a b}{2 \sigma} t-\left[\frac{1}{2 \sigma} \sqrt{(a-\sigma)^{2}+\frac{4 \mu \sigma}{\gamma(t)^{2}}}+\frac{1}{2}\right] \log (\beta)\right) \\
& =\lim _{t \rightarrow+\infty} \exp \left(\frac{a b}{2 \sigma} t-\left[\frac{1}{2 \sigma} \sqrt{(a-\sigma)^{2}+\frac{4 \mu \sigma}{\gamma(t)^{2}}}+\frac{1}{2}\right] b t\right) .
\end{aligned}
$$

- For $a>\sigma$, we have

$$
\begin{aligned}
\lim _{t \rightarrow+\infty} \mathbb{E}_{x}\left(e^{-\frac{\mu}{\gamma(t)^{2}} \int_{0}^{t} \frac{d s}{X_{s}}}\right) & =\lim _{t \rightarrow+\infty} \exp \left(\frac{a b}{2 \sigma} t-\left[\frac{a-\sigma}{2 \sigma} \sqrt{1+\frac{4 \mu \sigma}{\gamma(t)^{2}(a-\sigma)^{2}}}+\frac{1}{2}\right] b t\right) \\
& =\lim _{t \rightarrow+\infty} \exp \left(\frac{\mu b t}{\gamma(t)^{2}(a-\sigma)}+\frac{t}{\gamma(t)^{2}} \varepsilon\left(\frac{1}{\gamma(t)^{2}}\right)\right) .
\end{aligned}
$$

Taking $\gamma(t)^{2}=t$, we deduce that $\frac{1}{t} \int_{0}^{t} \frac{d s}{X_{s}}$ converges in distribution and of course in probability to the constant $b /(a-\sigma)$.

- For $a=\sigma$, we have

$$
\lim _{t \rightarrow+\infty} \mathbb{E}_{x}\left(e^{-\frac{\mu}{\gamma(t)^{2}} \int_{0}^{t} \frac{d s}{X_{s}}}\right)=\lim _{t \rightarrow+\infty} \exp \left(-\frac{b t}{\sqrt{\sigma} \gamma(t)} \sqrt{\mu}\right)
$$

Taking now $\gamma(t)=t$, we deduce that $\frac{1}{t^{2}} \int_{0}^{t} \frac{d s}{X_{s}}$ converges in distribution to $\tau_{2}$.

It remains now to prove the last assertion. For $b<0$, when $t$ goes to infinity in relation (9), since $\alpha$ converges to 0 and $\beta$ to $-b x / \sigma$, we have

$$
\begin{aligned}
\lim _{t \rightarrow+\infty} \mathbb{E}_{x}\left(e^{-\mu \int_{0}^{t} \frac{d s}{X_{s}}}\right)= & \frac{\Gamma\left(k+\frac{\nu}{2}+\frac{1}{2}\right)}{\Gamma(\nu+1)}\left(\frac{-b x}{\sigma}\right)^{\frac{\nu}{2}+\frac{1}{2}} \frac{1}{x^{k}}{ }_{1} F_{1}\left(k+\frac{\nu}{2}+\frac{1}{2}, \nu+1, \frac{-b x}{\sigma}\right) \\
& \times \lim _{t \rightarrow+\infty} \exp \left(\frac{b}{2 \sigma}[a t+2 x]-k \log \alpha\right) \\
= & \frac{\Gamma\left(k+\frac{\nu}{2}+\frac{1}{2}\right)}{\Gamma(\nu+1)}\left(\frac{-b x}{\sigma}\right)^{\frac{\nu}{2}+\frac{1}{2}-k} \exp \left(\frac{b x}{\sigma}\right){ }_{1} F_{1}\left(k+\frac{\nu}{2}+\frac{1}{2}, \nu+1, \frac{-b x}{\sigma}\right) .
\end{aligned}
$$

Finally, we conclude by identifying the limit distribution with relation (3) in Lemma 1. 


\section{Statistical Inference of the CIR model}

Let us first recall some basic notions on the construction of the maximum likelihood estimator (MLE). Suppose that the one dimensional diffusion process $\left(X_{t}\right)_{t \geq 0}$ satisfies

$$
d X_{t}=b\left(\theta, X_{t}\right) d t+\sigma\left(X_{t}\right) d W_{t}, \quad X_{0}=x_{0}
$$

where the parameter $\theta \in \Theta \subset \mathbb{R}^{p}, p \geq 1$, is to be estimated. The coefficients $b$ and $\sigma$ are two functions satisfying conditions that guarantee the existence and uniqueness of the SDE for each $\theta \in \Theta$. We denote by $\mathbb{P}_{\theta}$ the probability measure induced by the solution of the equation on the canonical space $C\left(\mathbb{R}_{+}, \mathbb{R}\right)$ with the natural filtration $\mathcal{F}_{t}:=\sigma\left(W_{s}, s \leq t\right)$, and let $\mathbb{P}_{\theta, t}:=\mathbb{P}_{\theta} \mid \mathcal{F}_{t}$ be the restriction of $\mathbb{P}_{\theta}$ to $\mathcal{F}_{t}$. If the integrals in the next formula below make sense then the measures $\mathbb{P}_{\theta, t}$ and $\mathbb{P}_{\theta_{0}, t}$, for any $\theta, \theta_{0} \in \Theta, t>0$, are equivalent (see Jacod [12] and Lipster and Shirayev [17]) and we are able to introduce the so called likelihood ratio

$$
L_{t}^{\theta, \theta_{0}}:=\frac{d \mathbb{P}_{\theta, t}}{d \mathbb{P}_{\theta_{0}, t}}=\exp \left\{\int_{0}^{t} \frac{b\left(\theta, X_{s}\right)-b\left(\theta_{0}, X_{s}\right)}{\sigma^{2}\left(X_{s}\right)} d X_{s}-\frac{1}{2} \int_{0}^{t} \frac{b^{2}\left(\theta, X_{s}\right)-b^{2}\left(\theta_{0}, X_{s}\right)}{\sigma^{2}\left(X_{s}\right)} d s\right\} .
$$

The process $\left(L_{t}^{\theta, \theta_{0}}\right)_{t \geq 0}$ is an $\mathcal{F}_{t}$-martingale.

In the present section, we observe the process $X^{T}=\left(X_{t}\right)_{0 \leq t \leq T}$ as a parametric model solution to equation (1), namely

$$
d X_{t}=\left(a-b X_{t}\right) d t+\sqrt{2 \sigma X_{t}} d W_{t}
$$

where $X_{0}=x>0, a>0, b \in \mathbb{R}, \sigma>0$. The unknown parameter, say $\theta$, is involved only in the drift part of the diffusion and we consider the two cases $\theta=b$ or $\theta=a$. This study includes both ergodic $(b>0$ and $a \geq \sigma)$ and nonergodic cases.

\subsection{Parameter estimation $\theta=b$}

The appropriate likelihood ratio (12), evaluated at time $T$ with $\theta_{0}=0$, is well defined and is given by

$$
L_{T}(b):=L_{T}^{\theta_{1} \theta_{0}}=\exp \left\{\frac{b}{2 \sigma}\left(x-X_{T}\right)+\frac{1}{4 \sigma} \int_{0}^{T}\left(2 a b-b^{2} X_{s}\right) d s\right\} .
$$

The MLE $\hat{b}_{T}$ of $b$ maximizes $L_{T}(b)$, then

$$
\hat{b}_{T}=\frac{a T+x-X_{T}}{\int_{0}^{T} X_{s} d s} .
$$

Hence, the error is given by

$$
\hat{b}_{T}-b=-\sqrt{2 \sigma} \frac{\int_{0}^{T} \sqrt{X_{s}} d W_{s}}{\int_{0}^{T} X_{s} d s} .
$$

As mentioned in the introduction, the above error is obviously of the form $M_{t} /\langle M\rangle_{t}$, where $\left(M_{t}\right)_{t \geq 0}$ is a Brownian martingale with quadratic variation $\langle M\rangle_{t}$. If this quadratic variation, 
correctly normalized, converges in probability then the classical martingale central limit theorem can be applied. Otherwise, the study of the couple $\left(X_{T}, \int_{0}^{T} X_{s}\right)$ will be helpful to investigate the limit law of the error. The asymptotic behavior of $\hat{b}_{T}-b$ can be summarized as follows.

Theorem 1 The MLE of b satisfies

1. Case $b>0: \mathcal{L}_{b}\left\{\sqrt{T}\left(\hat{b}_{T}-b\right)\right\} \Longrightarrow \mathcal{N}\left(0,2 \sigma \frac{b}{a}\right)$.

2. Case $b=0: \mathcal{L}_{b}\left\{T\left(\hat{b}_{T}-b\right)\right\} \Longrightarrow \frac{a-R_{1}}{I_{1}}$, where $\left(R_{t}\right)$ is the CIR process, starting from 0 , solution to (2) and $I_{t}=\int_{0}^{t} R_{s} d s$.

3. Case $b<0: \mathcal{L}_{b}\left\{e^{-b T / 2}\left(\hat{b}_{T}-b\right)\right\} \Longrightarrow \frac{G}{R}$, where $(G, R)$ is a couple of random variable characterized with its joint moment generating-Laplace transform. For $\lambda \in \mathbb{R}$ and $\mu \geq 0$,

$$
\mathbb{E}\left(e^{\lambda G-\mu R}\right)=\left(\frac{b}{\mu \sigma / b+b}\right)^{\frac{a}{\sigma}} \exp \left(x \frac{\sigma \lambda^{2} / b+\mu}{\mu \sigma / b+b}\right) .
$$

Therefore $G$ and $R$ are correlated, $G$ is normal and $R$ has the same distribution as $t_{0} R_{t_{0}}$, $t_{0}=-1 / b$, where $\left(R_{t}\right)_{t \geq 0}$ is the CIR process, starting from $x$, solution to (2).

Proof : In the case $b>0$, by Proposition 3 and the central limit theorem given by Y.A. Kutoyants (see Theorem 1.19 in [15]) we have

$$
\mathbb{P}_{b}-\lim _{T \rightarrow \infty} \frac{1}{T} \int_{0}^{T} X_{s} d s=\frac{a}{b}, \quad \mathcal{L}_{b}\left\{\frac{1}{\sqrt{T}} \int_{0}^{T} \sqrt{X_{s}} d W_{s}\right\} \Longrightarrow \mathcal{N}\left(0, \frac{a}{b}\right) .
$$

Therefore we obtain the first assertion. In the second case $b=0$, by Proposition 1 we have

$$
\mathcal{L}_{0}\left\{\frac{X_{T}}{T}, \frac{1}{T^{2}} \int_{0}^{T} X_{s} d s\right\} \Longrightarrow\left(R_{1}, I_{1}\right)
$$

Hence

$$
\mathcal{L}_{0}\left\{T\left(\hat{b}_{T}-b\right)\right\}=\mathcal{L}_{0}\left\{\frac{a+\frac{x}{T}-\frac{X_{T}}{T}}{\frac{1}{T^{2}} \int_{0}^{T} X_{s} d s}\right\} \Longrightarrow \frac{a-R_{1}}{I_{1}} .
$$

For the last case $b<0$, by Proposition 3 we have

$$
\mathcal{L}_{b}\left\{e^{b T} X_{T}, e^{b T} \int_{0}^{T} X_{s} d s\right\} \Longrightarrow\left(\tilde{R}_{t_{0}}, t_{0} \tilde{R}_{t_{0}}\right)
$$

where $t_{0}=-1 / b$ and $\left(\tilde{R}_{t}\right)_{t \geq 0}$ is the CIR process, starting from $x$, solution to (2). It follows that

$$
\mathcal{L}_{b}\left\{\hat{b}_{T}-b\right\}=\mathcal{L}_{b}\left\{\frac{e^{b T}\left(a T+x-X_{T}-b \int_{0}^{T} X_{s} d s\right)}{e^{b T} \int_{0}^{T} X_{s} d s}\right\} \Longrightarrow 0
$$


and also in probability, which proves the consistency of $\hat{b}_{T}$. Now, in order to study the asymptotic behavior of $\hat{b}_{T}-b$, we introduce the joint moment generating-Laplace transform of the renormalized couple $\left(e^{b T / 2}\left(-X_{T}-b \int_{0}^{T} X_{s} d s\right), e^{b T} \int_{0}^{T} X_{s} d s\right)$, namely for $\lambda$ in the neighborhood of the origin and $\mu \geq 0$ we consider

$$
\mathbb{E}_{x}\left(e^{-\lambda e^{b T / 2}\left(X_{T}+b \int_{0}^{T} X_{s} d s\right)-\mu e^{b T} \int_{0}^{T} X_{s} d s}\right)=\mathbb{E}_{x}\left(e^{-\lambda e^{b T / 2} X_{T}-\left(\lambda e^{b T / 2} b+\mu e^{b T}\right) \int_{0}^{T} X_{s} d s}\right) .
$$

By noting that the result of the first assertion in Lemma 2 remains valid for small values of $\lambda$, the above quantity becomes equal to $e^{-a \bar{\phi}_{\lambda, \mu}(T)} e^{-x \bar{\psi}_{\lambda, \mu}(T)}$ where

$$
\bar{\phi}_{\lambda, \mu}(T)=-\frac{1}{\sigma} \log \left(\frac{2 \rho e^{T(b+\rho) / 2}}{2 \sigma \lambda\left(e^{\left(\rho+\frac{b}{2}\right) T}-e^{\frac{b}{2} T}\right)+(\rho-b)+(\rho+b) e^{\rho T}}\right)
$$

and

$$
\bar{\psi}_{\lambda, \mu}(T)=\frac{\lambda e^{b T / 2}\left((\rho+b)+(\rho-b) e^{\rho T}\right)+2\left(\lambda e^{b T / 2} b+\mu e^{b T}\right)\left(e^{\rho T}-1\right)}{2 \sigma \lambda\left(e^{\left(\rho+\frac{b}{2}\right) T}-e^{\frac{b}{2} T}\right)+(\rho-b)+(\rho+b) e^{\rho T}},
$$

with $\rho=\sqrt{b^{2}+4 \sigma\left(\lambda e^{b T / 2} b+\mu e^{b T}\right)}$. Since $\rho$ goes to $-b$ and $(\rho+b) T$ goes to zero as $T$ tends to infinity, we have

$$
\lim _{T \rightarrow \infty} \bar{\phi}_{\lambda, \mu}(T)=-\frac{1}{\sigma} \lim _{T \rightarrow \infty} \log \left(\frac{-2 b}{\left(2 \sigma \lambda e^{\frac{b}{2} T}+\rho+b\right) e^{\rho T}-2 b}\right)
$$

and

$$
\lim _{T \rightarrow \infty} \bar{\psi}_{\lambda, \mu}(T)=\lim _{T \rightarrow \infty} \frac{\lambda e^{-b T / 2}(\rho+b)+2 \mu}{\left(2 \sigma \lambda e^{\frac{b}{2} T}+\rho+b\right) e^{\rho T}-2 b} .
$$

A Taylor's expansion gives $\lim _{T \rightarrow \infty}\left(2 \sigma \lambda e^{\frac{b}{2} T}+\rho+b\right) e^{\rho T}=-2 \sigma \mu / b$ and $\lim _{T \rightarrow \infty} e^{-b T / 2}(\rho+b)=$ $2 \sigma \lambda / b$. This completes the proof.

\subsection{Parameter estimation $\theta=a$}

According to relation (12), the appropriate likelihood ratio, evaluated at time $T$ with $\theta_{0}=0$, makes sense when $\mathbb{P}_{a}\left(\int_{0}^{T} \frac{d s}{X_{s}}<\infty\right)=1$ and is given by

$$
L_{T}(a):=L_{T}^{\theta, \theta_{0}}=\exp \left\{\frac{a}{2 \sigma} \int_{0}^{T} \frac{d X_{s}}{X_{s}}-\frac{1}{4 \sigma} \int_{0}^{T} \frac{a^{2}-2 a b X_{s}}{X_{s}} d s\right\} .
$$

The MLE $\hat{a}_{T}$ of a maximizes $L_{T}(a)$, then

$$
\hat{a}_{T}=\frac{b T+\int_{0}^{T} \frac{d X_{s}}{X_{s}}}{\int_{0}^{T} \frac{d s}{X_{s}}} .
$$

Hence, the error is given by

$$
\hat{a}_{T}-a=\sqrt{2 \sigma} \frac{\int_{0}^{T} \frac{d W_{s}}{\sqrt{X_{s}}}}{\int_{0}^{T} \frac{d s}{X_{s}}} .
$$


As explained in the last subsection, we can apply the classical martingale central limit theorem when $\int_{0}^{T} \frac{d s}{X_{s}}$, correctly normalized, converges in probability. Otherwise, using Itô's formula, we rewrite the MLE as follows

$$
\hat{a}_{T}=\frac{\log X_{T}-\log x+b T+\sigma \int_{0}^{T} \frac{d s}{X_{s}}}{\int_{0}^{T} \frac{d s}{X_{s}}}
$$

and now we study the couple $\left(\log \left(X_{T}\right), \int_{0}^{T} \frac{d s}{X_{s}}\right)$ in order to obtain a limit law for the error. The asymptotic behavior of $\hat{a}_{T}-a$ can be summarized as follows.

Theorem 2 The MLE of $a$ is well defined for $a \geq \sigma$ and satisfies

1. Case $b=0$ and $a>\sigma: \mathcal{L}_{a}\left\{\sqrt{\log T}\left(\hat{a}_{T}-a\right)\right\} \Longrightarrow \mathcal{N}(0,2 \sigma(a-\sigma))$.

2. Case $b>0$ and $a>\sigma: \mathcal{L}_{a}\left\{\sqrt{T}\left(\hat{a}_{T}-a\right)\right\} \Longrightarrow \mathcal{N}\left(0, \frac{2 \sigma(a-\sigma)}{b}\right)$.

3. Case $b=0$ and $a=\sigma: \mathcal{L}_{a}\left\{(\log T)\left(\hat{a}_{T}-a\right)\right\} \Longrightarrow \frac{1}{\tau_{1}}$, where $\tau_{1}$ is the hitting time associated with Brownian motion $\tau_{1}=\inf \left\{t>0: W_{t}=\frac{1}{\sqrt{2 \sigma}}\right\}$.

4. Case $b>0$ and $a=\sigma: \mathcal{L}_{a}\left\{T\left(\hat{a}_{T}-a\right)\right\} \Longrightarrow \frac{b}{\tau_{2}}$, where $\tau_{2}$ is the hitting time associated with Brownian motion $\tau_{2}=\inf \left\{t>0: W_{t}=\frac{b}{\sqrt{2 \sigma}}\right\}$.

5. Case $b<0$ and $a \geq \sigma$ : the MLE estimator $\hat{a}_{T}$ is not consistent.

Proof : At first, from Proposition 2 and 4, we have $\mathbb{P}_{a}\left(\int_{0}^{T} \frac{d s}{X_{s}}<\infty\right)=1$ for $a \geq \sigma$.

In the case $b=0$ and $a>\sigma$, by Proposition 2 and the central limit theorem, we have

$$
\mathbb{P}_{a}-\lim _{T \rightarrow \infty} \frac{1}{\log T} \int_{0}^{T} \frac{d s}{X_{s}}=\frac{1}{a-\sigma}, \quad \mathcal{L}_{a}\left\{\frac{1}{\sqrt{\log T}} \int_{0}^{T} \frac{d W_{s}}{\sqrt{X_{s}}}\right\} \Longrightarrow \mathcal{N}\left(0, \frac{1}{a-\sigma}\right) .
$$

This establishes the first assertion. The second one is obtained in the same manner using Proposition 4.

For the third case $b=0$ and $a=\sigma$, relation (14) yields

$$
\hat{a}_{T}-a=\frac{\log X_{T}-\log x}{\int_{0}^{T} \frac{d s}{X_{s}}} .
$$

The task is now to find the asymptotic behavior of $\log X_{T}$. By a scaling argument the process $\left(X_{2 t / a}\right)$ has the same distribution as a bidimensional square Bessel process, starting from $x$, $\mathrm{BESQ}_{x}^{2}$. It follows that

$$
X_{2 T / a} \stackrel{\text { law }}{=}\left\|B_{T}+x\right\|^{2} \stackrel{\text { law }}{=} T\left\|B_{1}+x / \sqrt{T}\right\|^{2},
$$


where $\left(B_{t}\right)_{t \geq 0}$ denotes a standard bidimensional Brownian motion. Hence, $\log X_{T} / \log T$ converges in law to one and consequently in probability. This gives us the announced result.

For the case $b>0$ and $a=\sigma$, as in the above case we can rewrite the error

$$
\hat{a}_{T}-a=\frac{\log X_{T}-\log x+b T}{\int_{0}^{T} \frac{d s}{X_{s}}} .
$$

Now, the CIR process $\left(X_{t}\right)$ can be represented in terms of a BESQ ${ }_{x}^{2}$ as follows

$$
X_{T} \stackrel{\text { law }}{=} e^{-b T} \operatorname{BESQ}_{x}^{2}\left(\frac{a}{2 b}\left(e^{b T}-1\right)\right) .
$$

Since $\log \mathrm{BESQ}_{x}^{2}(T) / \log T$ converges in distribution to one, we deduce that $\left(\log X_{T}-\log x+\right.$ $b T) / T$ converges in distribution to $b$ and consequently in probability. This gives our claim.

The only point remaining concerns the last case, $b<0$ and $a \geq \sigma$. On the one hand, according to the above representation in law of the CIR process as a time changed BESQ ${ }_{x}^{2}$, we have the convergence in distribution of $\log X_{T}+b T$ to the logarithm of $\operatorname{BESQ}_{x}^{2}(-a / 2 b)$. On the other hand, since $\left(\int_{0}^{t} \frac{d s}{X_{s}}\right)_{t \geq 0}$ is an increasing process, we deduce the almost surely convergence in the last assertion of Proposition 4. This completes the proof.

\section{Numerical Simulations}

Our aim in this section is to illustrate and test the practical behavior of the estimators errors stated above. For this purpose, we need to generate at time $T>0$, the CIR $X_{T}$, the so called Lévy area $\int_{0}^{T} X_{s} d s$ and $\int_{0}^{T} \frac{d s}{X_{s}}$.

One way to do that is to use numerical schemes solving the SDE, like the famous Euler scheme. However, any discretization scheme introduces bias into the simulation results; an extensive discussion on this subject is given by Kloeden and Platen [14]. Nevertheless, it is important to note that the discretization of the the CIR process present some troubles because of the square root in the diffusion coefficient. Several papers deal with this problem, see for example Alfonsi [1], Berkaoui, Bossy and Diop [2] and Deelstra and Delbaen [6]. However, the proposed schemes do not cover the nonergodic case in all its generality.

In the other hand, it is well known that the CIR $X_{T}$ is a non-central chi-squared random variable that can be simulated exactly. Therefore, Broadie and Kaya [3] propose an exact simulation of $\left(X_{T}, \int_{0}^{T} X_{s} d s\right)$ after they compute the conditional Laplace transform of $\int_{0}^{T} X_{s} d s$ given $X_{T}$. In our context, we proceed analogously for the simulation of $\left(X_{T}, \int_{0}^{T} \frac{d s}{X_{s}}\right)$. For this aim, we have to explicit also the conditional Laplace transform of $\int_{0}^{T} \frac{d s}{X_{s}}$ given $X_{T}$. This result is established in the subsection below to be used then in simulation.

\subsection{On The Conditional Law of $\int_{0}^{t} X_{s} d s$ and $\int_{0}^{t} \frac{d s}{X_{s}}$ Given $X_{t}$}

First, we give the conditional Laplace transform for the couple $\left(\int_{0}^{t} X_{s} d s, \int_{0}^{t} \frac{d s}{X_{s}}\right)$ given $X_{t}, t>0$, where $\left(X_{t}\right)_{t \geq 0}$ denotes a BESQ ${ }_{x}^{a}$ process, starting from $x$, solution to (2) with $\sigma=2$, namely $d X_{t}=a d t+2 \sqrt{X_{t}} d W_{t}$. 
Theorem 3 For the square Bessel process introduced above, we have

$$
\begin{aligned}
\mathbb{E}_{x}\left(e^{-\lambda \int_{0}^{t} X_{s} d s-\mu \int_{0}^{t} \frac{d s}{X_{s}}} \mid X_{t}=y\right)=\frac{\sqrt{2 \lambda} t}{\sinh (\sqrt{2 \lambda} t)} \exp \left(\frac{x+y}{2 t}[1-\sqrt{2 \lambda} t \operatorname{coth}(\sqrt{2 \lambda} t)]\right) \\
\times \frac{I_{\gamma}(\sqrt{2 \lambda x y} / \sinh (\sqrt{2 \lambda} t))}{I_{\nu}(\sqrt{x y} / t)}
\end{aligned}
$$

for all $\lambda \geq 0$ and $\mu>0$, with $\gamma=\sqrt{(a-2)^{2}+8 \mu} / 2$ and $\nu=a / 2-1$.

Proof On the one hand, by taking $\sigma=2$ in equation (4), we deduce that the fundamental solution to the PDE $u_{t}=2 x u_{x x}+a u_{x}-\left(\lambda x+\frac{\mu}{x}\right) u$, satisfying the relation $\mathbb{E}_{x}\left(e^{-\lambda \int_{0}^{t} X_{s} d s-\mu \int_{0}^{t} \frac{d s}{X_{s}}}\right)=$ $\int_{0}^{+\infty} p(t, x, y) d y$, is given by

$$
p(t, x, y)=\frac{\sqrt{2 \lambda}}{2 \sinh (\sqrt{2 \lambda} t)}\left(\frac{y}{x}\right)^{\nu / 2} \exp \left(-\frac{\sqrt{2 \lambda}}{2}(x+y) \operatorname{coth}(\sqrt{2 \lambda} t)\right) I_{\gamma}(\sqrt{2 \lambda x y} / \sinh (\sqrt{2 \lambda} t)) .
$$

On the other hand, by conditioning we rewrite

$$
\mathbb{E}_{x}\left(e^{-\lambda \int_{0}^{t} X_{s} d s-\mu \int_{0}^{t} \frac{d s}{X_{s}}}\right)=\int_{0}^{\infty} \mathbb{E}_{x}\left(e^{-\lambda \int_{0}^{t} X_{s} d s-\mu \int_{0}^{t} \frac{d s}{X_{s}}} \mid X_{t}=y\right) p_{X_{t}}(y) d y,
$$

where $p_{X_{t}}=\frac{1}{2 t}\left(\frac{y}{x}\right)^{\nu / 2} \exp \left(\frac{x+y}{2 t}\right) I_{\nu}(\sqrt{x y} / t)$ is the density of the Bessel process starting from $x$. The result follows by identification.

Remarks Here we comment the above result, in order to situate it and compare it with some known ones.

- From a probabilistic point of view, since we compute the conditional law of the couple instead of the marginal ones, Theorem 3 extend both formulas (6.5.2) and (6.5.3) of Proposition 6.5.1.1 p. 373, stated for $a \geq 2$, in Jeanblanc, Yor and Chesney [13].

- It is worth to note that, in the case $a<2$, when $\mu$ tends to 0 , we do not track down the marginal Laplace transform, since $\int_{0}^{t} \frac{d s}{X_{s}}$ is not finite almost surely. Indeed, in the general case $a>0$, this marginal Laplace transform is given by formula (2.m) page 432 of Pitman and Yor [19], namely

$$
\begin{aligned}
\mathbb{E}_{x}\left(e^{-\lambda \int_{0}^{t} X_{s} d s} \mid X_{t}=y\right)=\frac{\sqrt{2 \lambda} t}{\sinh (\sqrt{2 \lambda} t)} \exp \left(\frac{x+y}{2 t}[1\right. & -\sqrt{2 \lambda} t \operatorname{coth}(\sqrt{2 \lambda} t)]) \\
& \times \frac{I_{\nu}(\sqrt{2 \lambda x y} / \sinh (\sqrt{2 \lambda} t))}{I_{\nu}(\sqrt{x y} / t)}
\end{aligned}
$$

for all $\lambda \geq 0$, with $\nu=a / 2-1$. 
Now, we return to the general CIR process, starting from $x$, solution to (1), namely $d X_{t}=$ $\left(a-b X_{t}\right) d t+\sqrt{2 \sigma X_{t}} d W_{t}$ and we use the above result, to derive the following conditional laws.

Theorem 4 For a CIR process solution to (1), on the one hand, we have for $\lambda \geq 0$

$$
\begin{aligned}
& \mathbb{E}_{x}\left(e^{-\lambda \int_{0}^{t} X_{s} d s} \mid X_{t}=y\right)=\frac{\gamma(\lambda)}{b} \frac{\sinh (b t / 2)}{\sinh (\gamma(\lambda) t / 2)} \\
& \quad \times \exp \left(\frac{x+y}{2 \sigma}[b \operatorname{coth}(b t / 2)-\gamma(\lambda) \operatorname{coth}(\gamma(\lambda) t / 2)]\right) \frac{I_{\nu}\left(\frac{\gamma(\lambda)}{\sigma} \sqrt{x y} / \sinh (\gamma(\lambda) t / 2)\right)}{I_{\nu}\left(\frac{b}{\sigma} \sqrt{x y} / \sinh (b t / 2)\right)},
\end{aligned}
$$

with $\gamma(\lambda)=\sqrt{b^{2}+4 \lambda \sigma}$ and $\nu=\frac{a}{\sigma}-1$. On the other hand, we have for $\lambda>0$

$$
\mathbb{E}_{x}\left(e^{-\lambda \int_{0}^{t} \frac{d s}{X_{s}}} \mid X_{t}=y\right)=\frac{I_{\gamma(\lambda)}\left(\frac{b}{\sigma} \sqrt{x y} / \sinh (b t / 2)\right)}{I_{\nu}\left(\frac{b}{\sigma} \sqrt{x y} / \sinh (b t / 2)\right)}
$$

with $\gamma(\lambda)=\sqrt{(a-\sigma)^{2}+4 \lambda \sigma} / \sigma$ and $\nu=\frac{a}{\sigma}-1$

Proof Let us define the process $Y_{t}=X_{2 t / \sigma}$, by a scaling argument, we have

$$
d Y_{t}=\left(\alpha-\beta Y_{t}\right) d t+2 \sqrt{Y_{t}} d W_{t}, \quad Y_{0}=x,
$$

with $\alpha=\frac{2 a}{\sigma}$ and $\beta=\frac{2 b}{\sigma}$. We now write the Laplace transform

$$
\mathbb{E}_{x}\left(\exp \left(-\lambda \int_{0}^{t} \frac{d s}{X_{s}}\right) \mid X_{t}=y\right)=\mathbb{E}_{x}\left(\exp \left(-\frac{2 \lambda}{\sigma} \int_{0}^{\sigma t / 2} \frac{d s}{Y_{s}}\right) \mid Y_{2 t / \sigma}=y\right) .
$$

Thanks to the change of law formula (6.d) of Pitman and Yor [19], we get

$$
\mathbb{E}_{x}\left(\exp \left(-\lambda \int_{0}^{t} \frac{d s}{X_{s}}\right) \mid X_{t}=y\right)=\frac{\mathbb{E}_{x}\left(\exp \left(-\frac{2 \lambda}{\sigma} \int_{0}^{\sigma t / 2} \frac{d s}{R_{s}}-\frac{b^{2}}{2 \sigma^{2}} \int_{0}^{\sigma t / 2} R_{s} d s\right) \mid R_{2 t / \sigma}=y\right)}{\mathbb{E}_{x}\left(\exp \left(-\frac{b^{2}}{2 \sigma^{2}} \int_{0}^{\sigma t / 2} R_{s} d s\right) \mid R_{2 t / \sigma}=y\right)}
$$

where $\left(R_{t}\right)_{t \geq 0}$ is a $\mathrm{BESQ}_{x}^{2 a / \sigma}$ process. In the same manner

$$
\mathbb{E}_{x}\left(\exp \left(-\lambda \int_{0}^{t} X_{s} d s\right) \mid X_{t}=y\right)=\frac{\mathbb{E}_{x}\left(\exp \left(-\left(\frac{2 \lambda}{\sigma}+\frac{b^{2}}{2 \sigma^{2}}\right) \int_{0}^{\sigma t / 2} R_{s} d s\right) \mid R_{2 t / \sigma}=y\right)}{\mathbb{E}_{x}\left(\exp \left(-\frac{b^{2}}{2 \sigma^{2}} \int_{0}^{\sigma t / 2} R_{s} d s\right) \mid R_{2 t / \sigma}=y\right)}
$$

We complete the proof, by combining relations (15) and (16) with the last two equations. 


\subsection{Numerical Results}

The task now is to check the validity of the results obtained on our estimators and to understand how fast the convergence actually takes place as $T \rightarrow \infty$, with computer simulations. By relation (13), the illustrations of Theorem 1, about the MLE of $b$, involve the simulation of the couple $\left(X_{T}, \int_{0}^{T} X_{s} d s\right)$. Therefore, we use the exact simulation method proposed by Broadie and Kaya [3] to generate it. For Theorem 2, concerning the MLE of $a$, we use relation (14) and we introduce a new exact simulation method for the couple $\left(X_{T}, \int_{0}^{T} \frac{d s}{X_{s}}\right)$ based on the theoretical results of the above subsection.

\subsubsection{The MLE of $b$}

The method of Broadie and Kaya [3] is to simulate, at first, the random variable $X_{T}$ with a noncentral chi-squared distribution

$$
X_{T} \stackrel{l a w}{=} \frac{\sigma\left(1-e^{-b t}\right)}{2 b} \chi_{\frac{2 a}{\sigma}}^{\prime 2}\left(\frac{2 b e^{-b t}}{\sigma\left(1-e^{-b t}\right)} x\right), \quad t>0
$$

where $\chi_{d}^{\prime 2}(n c)$ denotes the noncentral chi-squared random variable with $d$ degrees of freedom and noncentrality parameter $n c$. The second step is to deduce the conditional characteristic function, say $\Phi$, of $\int_{0}^{T} X_{s} d s$ given $X_{T}$ by setting $\lambda=-i u, u \in \mathbb{R}$, in relation (17). Then, the cumulative distribution function $F$ of the conditional law is computed using Fourier inversion method. More precisely, we have

$$
F(x)=\frac{1}{\pi} \int_{-\infty}^{+\infty} \frac{\sin (u x)}{u} \Phi(u)=\frac{2}{\pi} \int_{0}^{+\infty} \frac{\sin (u x)}{u} \operatorname{Re}[\Phi(u)] d u .
$$

This integral is approximated, using a trapezoidal rule with step discretization $h$, by an infinite sum that is truncated to an order $N$, namely

$$
F(x) \simeq \frac{h x}{\pi}+\frac{2}{\pi} \sum_{j=1}^{N} \frac{\sin (h j x)}{j} \operatorname{Re}[\Phi(h j)] .
$$

The choice of parameters $h$ and $N$ to achieve a desired accuracy is well explained in their paper. Nevertheless, they draw attention to the continuity problem in the numerical representation of the modified Bessel function of first kind, with a complex argument, that appears in the numerator of the characteristic function $\Phi$. In fact, the modified Bessel function of first kind characterized by the following power series

$$
I_{\nu}(z)=\left(\frac{z}{2}\right)^{\nu} \sum_{j=0}^{\infty} \frac{\left(\frac{z^{2}}{4}\right)^{j}}{j ! \Gamma(\nu+j+1)}
$$

where $\Gamma(x)$ is the gamma function and $z$ is a complex number, presents a discontinuity problem in the representation of the power term $z^{\nu}$. Because this last function is multivalued and most of software packages consider it equal to $\exp (\nu \log (z))$, where $\log (z)$ is computed on the principal 
branch of $\arg (z)$. To avoid this difficulty, Broadie and Kaya carefully tracked $\arg (z)$ when evaluating $I_{\nu}(z)$ and changed the branch when necessary by $I_{\nu}\left(z e^{m \pi i}\right)=e^{m \nu \pi i} I_{\nu}(z)$, where $m$ is an integer value. Recently, Lord and Kahl [18] showed how to avoid this complex discontinuity problem. They considered, up to a scaling coefficient, the complex-valued argument in the modified Bessel function, $z(u)=\gamma(u) / \sinh (\gamma(u) t)$, where $\gamma(u)=\sqrt{b^{2}-4 \sigma i u}$ and evaluated the characteristic function as $\Phi(u) e^{\nu \log (z(u))} / z(u)^{\nu}$, where $\Phi(u)$ and $z(u)^{\nu}$ are evaluated using the principal branch, however, $\log (z(u))$ is evaluated as $-\gamma(u) t / 2+\log \left(\gamma(u) /\left(1-e^{\gamma(u) t}\right)\right)$, the last logarithm term is evaluated on its principal branch. This correction term introduced by Lord and Kahl [18] improves considerably our simulations.

The graphical representations of Figure 1 illustrate the limit law of the MLE $\hat{b}_{T}$ of $b$, stated in Theorem 1. In order to do that, we simulate $N$ independent trajectories of the normalized error, with different values of parameters $(x, a, b, c, T)$ to cover the various cases, and we plot their histogram. When the limit is Gaussian, we normalize the error by the appropriate term to compare the histogram with the standard Gaussian density. Otherwise, since we do not have an explicit formula of the limit law density, we simply plot the histogram of the error as stated in the theorem. Both histograms, on the top of Figure 1, deal with ergodic and nonergodic cases, for $b>0$. Those at the bottom, treat the cases $b=0$ and $b<0$. Note that in the latter cases, we choose $a<\sigma$ to cover the subtle case when the CIR can reaches the state 0 .
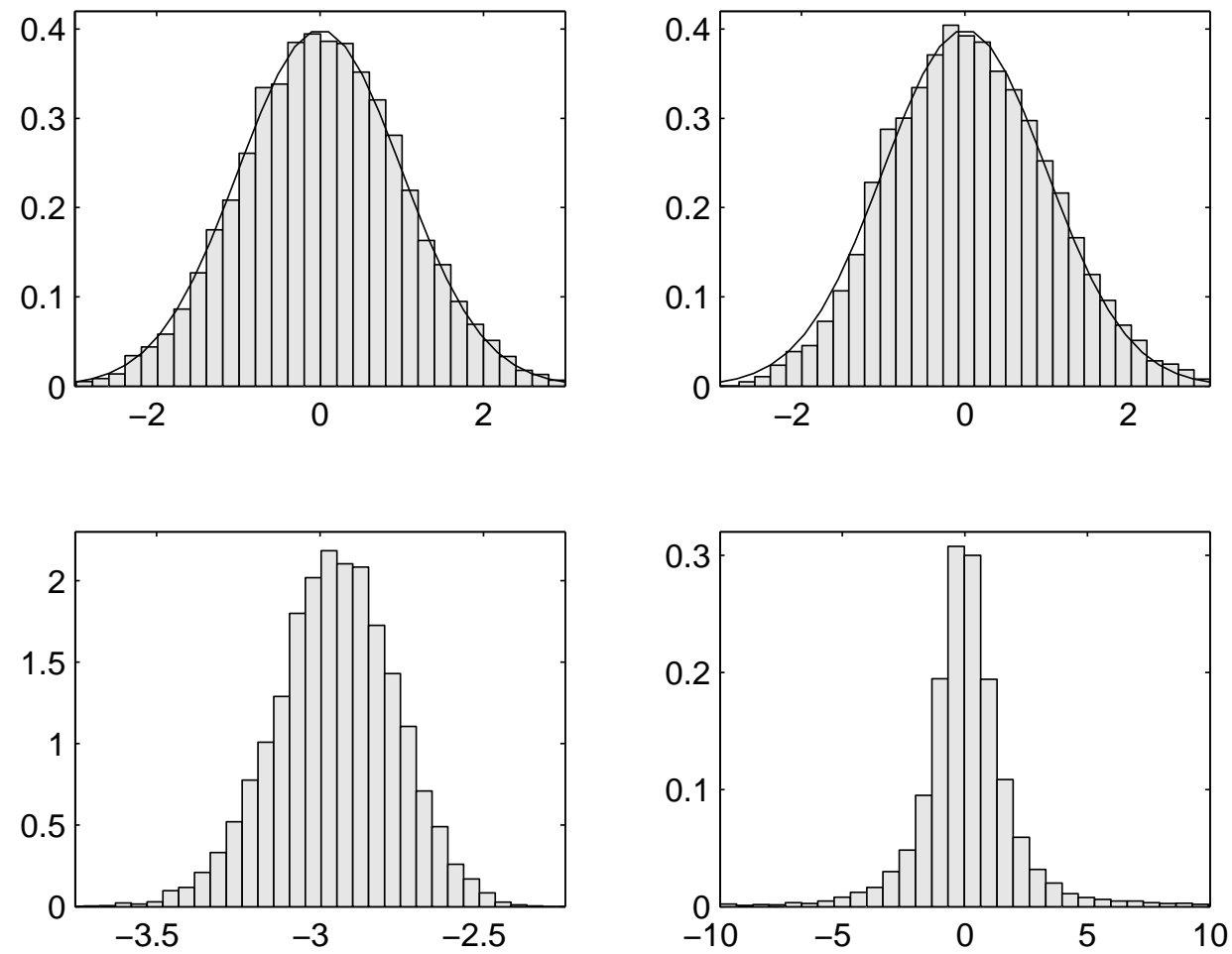

Figure 1: Convergence in distribution of the error, $\hat{b}_{T}-b$, correctly normalized, in different cases, for a sample $N=10000$, from the left to the right, we have $(x, a, b, \sigma, T)$ equal to $(1,2,1,1,1 e+3),(1,0.5,1,1,1 e+3),(1,0.75,0,1,1 e+2)$ and $(1,0.75,-1,1,12)$. 
Remark From a practical point of view, note that when the theoretical rate of convergence is of order $T$ (resp. $T^{2}$ ) the limit distribution is well approximated from $T=1 e+3(\operatorname{resp} . T=$ $1 e+2)$. However, when the theoretical rate of convergence is exponential then a stabilization is observed only from $T=12$ (See Figure 1).

\subsubsection{The MLE of $a$}

We proceed to simulate the couple $\left(X_{T}, \int_{0}^{T} \frac{d s}{X_{s}}\right)$ in the same manner as below. First, we begin by generating a random variable $X_{T}$ using the property (19). Then, in order to simulate $\int_{0}^{T} \frac{d s}{X_{s}}$, we use relation (18) to compute the cumulative distribution function of the conditional law. To do that, we use the Fourier inversion method introduced previously.

The graphical representation below illustrates the limit law of the MLE $\hat{a}_{T}$ of $a$ stated in Theorem 2. We simulate $N$ independent trajectories of the correctly normalized error, as explained in the subsection above, and we plot their histogram. Both histograms, on the top of Figure 2, deal with ergodic and nonergodic cases, for $a>\sigma$. Those at the bottom treat cases $b>0$ and $b=0$, for $a=\sigma$.
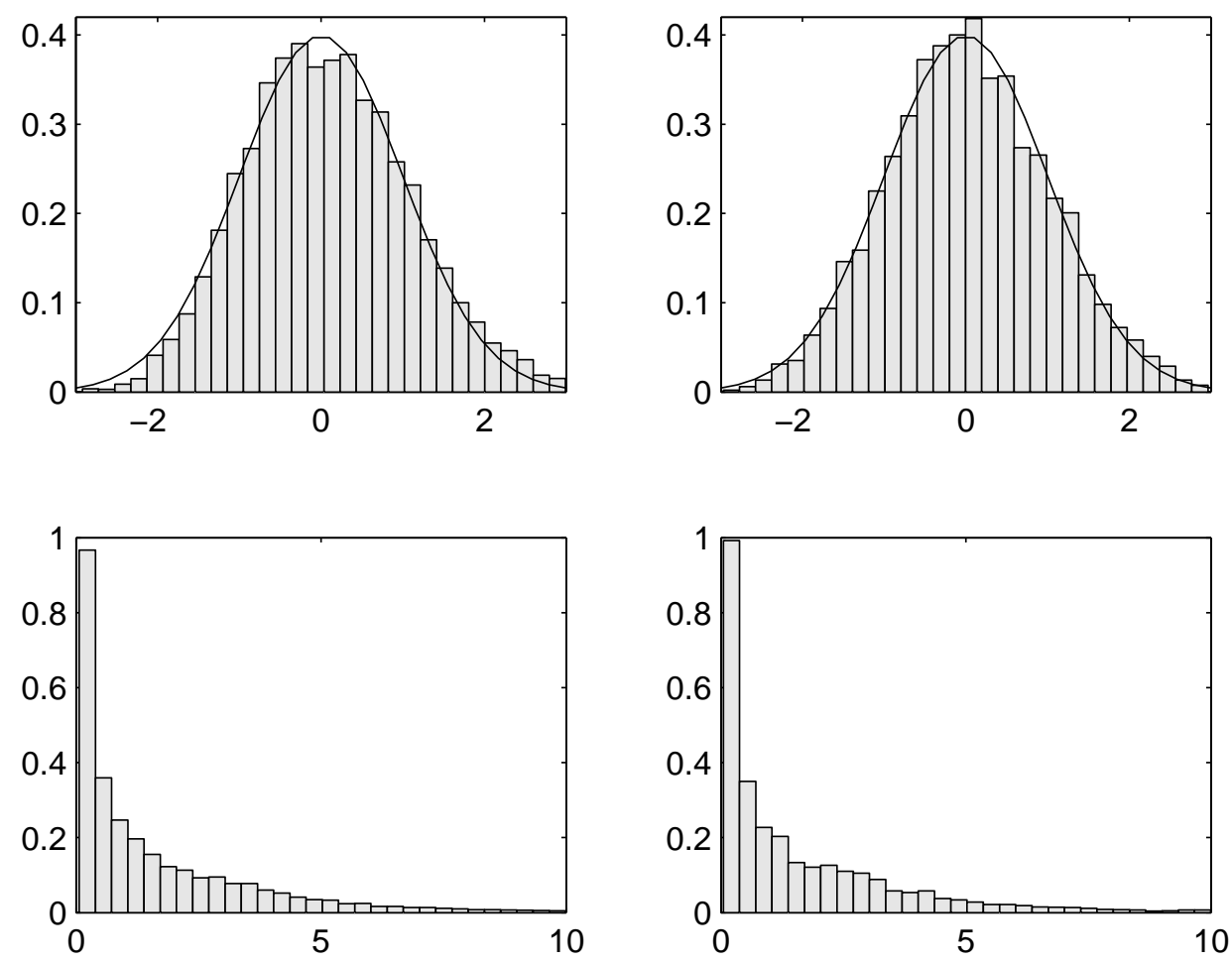

Figure 2: Convergence in distribution of the error, $\hat{a}_{T}-a$, correctly normalized, in different cases, for a sample $N=10000$, from the left to the right, we have $(x, a, b, \sigma, T)$ equal to $(1,2,0,1,1 e+100),(1,2,1,1,1 e+3),(1,1,0,1,1 e+50)$ and $(1,1,1,1,1 e+2)$. 
Remark In this case, note that when the theoretical rate of convergence is of order $\sqrt{T}$ (resp. $T)$ the limit distribution is well approximated from $T=1 e+3(\operatorname{resp} . T=1 e+2)$. However, when the theoretical rate of convergence is of order $\sqrt{\log T}(\operatorname{resp} . \log T)$ then to observe a stabilization $T=1 e+100$ (resp. $T=1 e+50$ ) was needed (See Figure 2).

\section{Conclusion}

The precise description of the behavior of $\int_{0}^{T} X_{s} d s$ and $\int_{0}^{T} \frac{d s}{X_{s}}$ established in the present paper provides a new approach to overcome the problem of parameters estimation for the CIR model in all its generality. When we estimate one of the drift parameter and suppose known the other one, we obtain original results that are confirmed by exact simulation methods. A natural question is now the problem of the global estimation for the CIR model. Answering this question involves more complicated calculations and this is the object of a forthcoming work.

\section{References}

[1] Aurélien Alfonsi. On the discretization schemes for the CIR (and Bessel squared) processes. Monte Carlo Methods Appl., 11(4):355-384, 2005.

[2] Abdel Berkaoui, Mireille Bossy, and Awa Diop. Euler scheme for SDEs with non-Lipschitz diffusion coefficient: strong convergence. ESAIM Probab. Stat., 12:1-11 (electronic), 2008.

[3] Mark Broadie and Özgür Kaya. Exact simulation of stochastic volatility and other affine jump diffusion processes. Oper. Res., 54(2):217-231, 2006.

[4] John C. Cox, Jonathan E. Ingersoll, Jr., and Stephen A. Ross. A theory of the term structure of interest rates. Econometrica, 53(2):385-407, 1985.

[5] Mark Craddock and Kelly A. Lennox. The calculation of expectations for classes of diffusion processes by Lie symmetry methods. Ann. Appl. Probab., 19(1):127-157, 2009.

[6] G. Deelstra and F. Delbaen. Convergence of discretized stochastic (interest rate) processes with stochastic drift term. Appl. Stochastic Models Data Anal., 14(1):77-84, 1998.

[7] Éric Fournié and Denis Talay. Application de la statistique des diffusions à un modèle de taux d'intérêt. Finance, 12(2):79-111, 1991.

[8] Anja Göing-Jaeschke and Marc Yor. A survey and some generalizations of Bessel processes. Bernoulli, 9(2):313-349, 2003.

[9] I. S. Gradshteyn and I. M. Ryzhik. Table of integrals, series, and products. Academic Press Inc., San Diego, CA, sixth edition, 2000. Translated from the Russian, Translation edited and with a preface by Alan Jeffrey and Daniel Zwillinger.

[10] S. L. Heston. A closed-form solution for options with stochastic volatility, with applications to bond and currency options. Rev. Finan. stud., 6(2):327-343, 1993. 
[11] Nobuyuki Ikeda and Shinzo Watanabe. Stochastic differential equations and diffusion processes, volume 24 of North-Holland Mathematical Library. North-Holland Publishing Co., Amsterdam, 1981.

[12] Jean Jacod. Inference for stochastic process. Technical Report, (638), 2001.

[13] Monique Jeanblanc, Marc Yor, and Marc Chesney. Mathematical methods for financial markets. Springer Finance. Springer-Verlag London Ltd., London, 2009.

[14] Peter E. Kloeden and Eckhard Platen. Numerical solution of stochastic differential equations, volume 23 of Applications of Mathematics (New York). Springer-Verlag, Berlin, 1992.

[15] Yury A. Kutoyants. Statistical inference for ergodic diffusion processes. Springer Series in Statistics. Springer-Verlag London Ltd., London, 2004.

[16] Damien Lamberton and Bernard Lapeyre. Introduction au calcul stochastique appliqué à la finance. Ellipses Édition Marketing, Paris, second edition, 1997.

[17] R. S. Liptser and A. N. Shiryayev. Statistics of random processes. I,II. Springer-Verlag, New York, 1977. General theory, Translated by A. B. Aries, Applications of Mathematics, Vol. 5.

[18] Roger Lord and Christian Kahl. Complex logarithms in Heston-like models. Math. Finance, 20(4):671-694, 2010.

[19] Jim Pitman and Marc Yor. A decomposition of Bessel bridges. Z. Wahrsch. Verw. Gebiete, 59(4):425-457, 1982.

[20] Daniel Revuz and Marc Yor. Continuous martingales and Brownian motion, volume 293 of Grundlehren der Mathematischen Wissenschaften [Fundamental Principles of Mathematical Sciences]. Springer-Verlag, Berlin, third edition, 1999. 